\section{Respostas hemodinâmicas durante exercício resistido com intensidades moderadas em idosas hipertensas}

\section{Hemodynamic responses during resistance exercise with moderate intensities in elderly hypertensive patients}

Aline de Freitas Brito ${ }^{1,2}$

Alexandre Sérgio Silva ${ }^{1,2}$

Maria do Socorro Brasileiro Santos ${ }^{1,3}$

Amilton da Cruz Santos ${ }^{1,3}$

\section{Resumo}

O objetivo desse estudo foi avaliar a resposta da frequência cardíaca (FC), pressão arterial e sua modulação autonômica durante no exercício resistido com diferentes intensidades em idosos hipertensos. Doze idosas hipertensas e oito idosas normotensas foram submetidas à uma sessão de exercício resistido na cadeira extensora à 40\% (EX40\%) e 60\% (EX60\%) de uma repetição máxima (1RM). FC, PA e a variabilidade da frequência cardíaca foram obtidas antes, durante e após as sessões de exercício. Diante disso, observou-se que os protocolos de EX40\% e EX60\% promoveram um aumento significativo da FC em ambos os grupos, e este comportamento foi acompanhado pelo aumento significativo no componente de baixa frequência $(\mathrm{BF})$ no balanço autonômico $(\mathrm{BF} / \mathrm{AF}) \mathrm{e}$ na redução da alta frequência $(\mathrm{AF})$ em relação à condição basal, para ambos os grupos. No período de recuperação, os componentes de $\mathrm{BF}, \mathrm{AF}$ e $\mathrm{BF} / \mathrm{AF}$ retornaram aos valores basais em ambos os grupos no protocolo $\mathrm{EX} 40 \%$. Em EX60\%, BF, AF e BF/AF foram restaurados a valores próximos dos basais para os hipertensos. Além disso, observou-se uma redução da Pressão arterial sistólica de $20 \mathrm{mmHg}$ para o grupo de normotensos e hipertensos no protocolo EX40\%. Ainda para a Pressão arterial sistólica, na intensidade EX60\%, os grupos normotensos e hipertenso apresentaram uma redução 20 e $21 \mathrm{mmHg}$, respectivamente. Conclui-se que o exercício resistido com intensidades moderadas não promove exacerbação da modulação autonômica cardíaca em idosos hipertensos durante sua execução, visto que sua resposta foi similar a aquela observada em normotensos.

\section{Palavras-chave}

Exercício; Sistema nervoso autônomo; Idosos.

\begin{abstract}
The aim of this study was to evaluate the response of heart rate (HR), blood pressure (BP) and its autonomic modulation during the resistance exercise with different intensities in elderly bypertensive patients. Twelve elderly hypertensive and eight normotensive elderly underwent a session of resistance exercise (RE) in knee extension at 40\% (EX40\%) and 60\% (EX60\%) of one repetition maximum $(1 R M) . H R, B P$ and heart rate variability were obtained before, during and after exercise sessions. Thus, it was observed that the protocols EX40\% and EX60\% promoted a significant increase in HR in both groups, and this behavior was accompanied by a significant increase in the low frequency component $(L F)$ in autonomic balance $(L F / H F)$ and reducing the high frequency $(H F)$ in relation to baseline values for both groups. During the recovery period, the components of $L F, H F$ and $L F / H F$ returned to baseline in both groups in the protocol EX40\%. In EX60\%, LF, HF and LF/HF were restored to values close to baseline for hypertensive patients. Furthermore, we observed a reduction in Systolic Blood Pressure of $20 \mathrm{mmHg}$ for the group of normotensive and hypertensive patients in the protocol EX40\%. Even for systolic blood pressure, intensity EX60\%, bypertensive and normotensive group showed a reduction 20:21 $\mathrm{mmHg}$, respectively. We conclude that resistance exercise with moderate intensity exercise does not promote exacerbation of cardiac autonomic modulation in elderly bypertensive patients during their execution, as their response was similar to that observed in normotensive.
\end{abstract}

\section{Keywords}

Exercise; Autonomic Nervous System; Aged.
Rev Bras Ativ Fis Saúde p. 464-474 DOI:

http://dx.doi.org/10.12820/rbafs.v.18n4p464

1 Departamento de Educação Física (DEF)/ Universidade Federal da Paraiba (UFPB); Paraiba-Brasil.

2 Laboratório de Estudos do Treinamento Físico Aplicado ao Desempenho e à Saúde DEF/UFPB; Paraiba- Brasil.

3 Grupo de Estudos do Treinamento Físico Aplicado à Saúde - DEF/UFPB; Paraíba-Brasil. 


\section{INTRODUÇÃO}

A disfunção autonômica cardíaca tem sido observada com o processo do envelhecimento ${ }^{1}$. Essas modificações se manifestam através da hiperatividade simpática, e da redução da atividade vagal, as quais, expõem os sujeitos à uma menor cardioproteção ${ }^{2}$, predispondo a arritmias cardíaca e/ou taquicardia ventricular sustenta$\mathrm{da}^{3}$, aumentando o risco de mortalidade por eventos cardíacos ${ }^{4}$.

Por outro lado, sabemos que o exercício resistido (ER) traz efeitos benéficos ao organismo humano. Alguns estudos têm demonstrado que a participação em programas de treinamento físico com ER restringe disfunções como, sarcopenia e atrofia muscular ${ }^{5}$, resistência à insulina ${ }^{6}$, elevação da pressão arterial ${ }^{7}$, entre outros benefícios. Diante destas evidências, o ER tornou-se comumente aceito e utilizado nos programas de treinamento físico para idoso ${ }^{8}$ estando as diretrizes que fundamentam sua prescrição consolidadas nas recomendações do American College of Sports Medicine e do American Heart Association, desde $2007{ }^{9}$.

Mesmo assim, poucos são os estudos que têm procurado investigar a participação da modulação autonômica cardíaca no $\mathrm{ER}^{10-11}$. Até onde sabemos, apenas o estudo de Takahashi et al., ${ }^{12}$ investigou o controle autonômico cardíaco em idosos, contudo, neste estudo, idosos saudáveis foram submetidos a exercício isométrico. Neste sentido, investigações quanto à segurança dos exercícios resistidos para idosos hipertensos ainda são necessárias, especialmente do ponto de vista da repercussão da modulação autonômica cardíaca.

Considerando que a associação entre a hipertensão arterial e o envelhecimento pode potencializar as desordens no mecanismo autonômico cardíaco, resolveuse verificar se a condição de hipertensão arterial afeta a resposta autonômica à exercícios resistidos. Sendo assim, o objetivo desse estudo foi avaliar a resposta da Frequência Cardíaca e sua modulação autonômica durante ER com intensidades leve e moderada em idosos hipertensos.

\section{MÉTODOS}

\section{Amostra}

O estudo foi desenvolvido com 20 idosas, participantes do programa de exercício físico para idosos do Departamento de Educação Física da Universidade Federal da Paraíba. Elas foram divididas em dois grupos: grupo hipertenso (66 \pm 3 anos, $\left.25 \pm 4 \mathrm{Kg} / \mathrm{m}^{2}\right)$, e normotenso $\left(64 \pm 1\right.$ anos, $\left.21 \pm 3 \mathrm{Kg} / \mathrm{m}^{2}\right)(\mathrm{p}<0,406)$, compostos respectivamente por doze e oito mulheres. De acordo com exames físicos e laudo médico apresentado pelas pacientes e conforme classificação proposta pelas VI Diretrizes da Sociedade Brasileira de Hipertensão ${ }^{13}$. A determinação do tamanho da amostra foi realizada utilizando o software Gpower 3.1.0 (Franz Faul, Universitat Kiel, Germany). Adotamos um poder estatístico de 0,90, um nível de confiança de 0,05 . Estimamos uma diferença de $122 \mathrm{bpm}$ para a freqüência cardíaca e um desvio padrão residual de $25 \mathrm{bpm}$, segundo o estudo de Lamotte et al., ${ }^{14}$. Como resultado, estimou-se um mínimo de oito sujeitos para compor cada grupo.

As voluntárias foram recrutadas de um Programa de Exercício Resistido da Academia de Ginástica da Universidade Federal da Paraíba. Para participar do estudo, as mulheres deveriam apresentar entre 60 e 70 anos, IMC inferior a 30 $\mathrm{kg} / \mathrm{m}^{2}$ e deveriam praticar exercícios resistidos regularmente há pelo menos seis meses (assiduidade de três ou mais vezes por semana). As voluntárias selecionadas para compor o grupo de hipertensas, apresentavam níveis pressóricos compatíveis 
que ao de grau leve, conforme classificação médica e deveriam utilizar apenas medicamentos anti-hipertensivos da classe dos inibidores da enzima conversora da angiotensina e/ou diuréticos. Todas mantiveram as medicações durante o estudo. Mulheres que apresentassem outras doenças crônicas como comorbidade não foram consideradas para o estudo.

Todos os sujeitos foram informados sobre os objetivos e procedimentos do estudo e ao aceitar participar do mesmo, assinaram o Termo de Consentimento Livre e Esclarecido, aprovado pelo Comitê de Ética e Pesquisa do Centro de Ciências da Saúde da Universidade Federal da Paraíba, (protocolo no 0259), de acordo com a Resolução n¹96/96 do Conselho Nacional de Saúde.

\section{Protocolo Experimental}

\section{- Sessão de familiarização e teste de carga máxima}

Na primeira semana, as idosas foram submetidas a uma sessão de adaptação aos exercícios de força. Nessa sessão, eles realizaram o mesmo exercício que seria utilizado na sessão experimental (exercício na cadeira extensora da marca Life Fitness, modelo 2000, com sobrecarga de cinco quilogramas) com a menor carga possível.

Setenta e duas horas pós, foi realizado o teste de uma repetição máxima (1RM) conforme proposta de Kraemer e Fry ${ }^{15}$. Previamente às sessões experimentais as voluntárias foram instruídas a não realizar exercícios físicos, não ingerir bebidas alcoólicas ou cafeinadas pelo menos 24 horas antes da sessão, além de manter uma rotina normal de utilização de medicação anti-hipertensiva.

\section{- Sessão Experimental}

$\mathrm{Na}$ semana seguinte foi realizada a sessão experimental, onde ao chegar ao laboratório, as voluntárias se deslocaram para a sala de experimento, onde foram instrumentadas, com um cinto envolvendo o quadril com a finalidade de estabilização na cadeira extensora, esfigmomanômetro de coluna de mercúrio no braço esquerdo (BD, Brasil), eletrodos para leitura do eletrocardiograma (System 1000 Modular Instrumentation, CWE-INC/USA), bem como a cinta respiratória (Pneumotrace, General Purpose Amplifier/Stemtech, Inc. GPA-4 model 2), ao final de 10 minutos de repouso, pressão arterial e a frequência cardíaca foram mensuradas. Ao final do período de repouso, os indivíduos foram submetidos ao protocolo de exercício. Nesse momento, a atividade autonômica cardíaca e a frequência cardíaca foram registradas durante todo o exercício, e a pressão arterial foi mensurada ao final deste. Após a intervenção, as voluntárias permaneceram sentadas na cadeira em repouso, por um período de 30 minutos, para nova mensuração da pressão arterial e frequência cardíaca.

\section{- Protocolo de Exercício Resistido}

As hipertensas foram submetidas a duas intensidades de ER com 40\% de 1RM (EX40\%) e com 60\% de 1RM (EX60\%). Em ambos, grupos do estudo, as idosas realizaram o exercício com 30 repetições, cadência de 2 s na fase excêntrica e concêntrica e intervalo de recuperação entre as sessões de 48 horas.

\section{Mensurações}

- Frequência cardíaca

Para determinação da freqüência cardíaca foi utilizado o eletrocardiógrafo. Foram 
colocados três eletrodos no tórax do paciente, na derivação DII. A aquisição e visualização do sinal do ECG foram obtidas por meio do software Windaq Aquisition (DATAQ Instruments - Akron, Oshio, USA).

\section{- Frequência respiratória}

A frequência respiratória foi obtida com a cinta respiratória, a qual contém sensores bilaterais que captam o sinal respiratório através da distensibilidade torácica. O sinal da respiração foi pré-amplificado (General Purpose Amplifier/Stemtech, Inc. GPA-4, modelo 2), e convertido de analógico para digital. Em tempo real, o sinal da respiração foi armazenado em computador através de programa Windaq DI200.

\section{- Pressão arterial}

As medidas foram realizadas no período pré-intervenção após 10 minutos de repouso, ao final do exercício e aos 30 minutos do período de recuperação. A medida foi sempre realizada pelo mesmo pesquisador, o qual já possuía habilidade de manuseio com o aparelho utilizado. O braço de aferição da Pressão Arterial (PA) foi mantido fixo nas duas sessões realizadas por cada indivíduo. A medida auscultatória da pressão arterial foi realizada no braço esquerdo com estetoscópio (Premium) e esfigmomanômetro de coluna de mercúrio devidamente calibrado (BD, Brasil).

\section{- Análise Espectral}

As voluntárias foram avaliadas antes, durante e após-intervenção. No momento de pré-intervenção, as idosas foram devidamente instrumentadas e repousaram por 10 minutos sentadas na cadeira extensora. Em seguida, a modulação autonômica foi medida durante toda execução do exercício, por um período em torno de três minutos. Após o exercício, ela foi novamente medida por um período de 30 minutos. Para medir a modulação autonômica cardíaca, os intervalos R-R do eletrocardiograma foram determinados, utilizando o software WINDAQ(DATAQ Instruments - Akron, Oshio, USA). A série temporal gerada (tacograma) foi analisada através do remodelamento matemático auto-regressivo, com um software desenvolvido no Departamento de Ciências Pré Clínica, Universitá Di Milano- Italy (Alberto Porta, Malliani, Montano, 1991). Assim, em trechos de pelo menos 150 ciclos cardíacos, o espectro foi gerados utilizando o recurso de Levinson-Durbin e a ordem do modelo foi escolhida de acordo com o critério de Akaike ${ }^{16}$. Foram gerados, então, os componentes de baixa (BF - 0,04 a 0,15 Hz) e alta $(\mathrm{AF}-0,15$ a $0,4 \mathrm{~Hz})$ frequências, que se relacionam, respectivamente, com as modulações, principalmente, simpática e parassimpática cardíacas. Os poderes de cada componente foram analisados em unidades normalizadas. A unidade normalizada foi calculada usando-se a potência de cada componente dividida pela potência total menos o componente de muito baixa frequência $(<0,04 \mathrm{~Hz})$. Adicionalmente, a razão BF/AF, um índice do balanço simpatovagal cardíaco, também foi calculada ${ }^{17}$.

\section{Análise Estatística}

A normalidade da variância dos dados foi verificada pelo teste de Shapiro-Wilk. Para a análise estatística, foi utilizado o programa estatístico: (STATISTICA para WINDOWS ver.4.3, StatsoftInc., 1993, Tulsa, OK, USA). As alterações nos parâmetros hemodinâmicos e autonômicos em cada sessão foram calculadas pela diferença entre os valores medidos nos períodos pós, durante e pré-intervenção. Para 
analisar o efeito das intensidades em todas as variáveis foi utilizado o ANOVA de três fatores para medidas repetidas, tendo como fatores principais a sessão e os tempos. O teste post hoc de Newman-keuls foi utilizado para localização das diferenças nas células quando adequado. Para todas as análises, o nível de significância adotado foi de $\mathrm{p}<0,05$. Os dados estão apresentados como média \pm desvio-padrão.

\section{RESULTADOS}

\section{Características dos sujeitos}

A tabela 1 apresenta as características antropométricas, hemodinâmicas, autonômicas basais, medicamentosas e a carga obtida no teste de 1RM nos grupos normotenso e hipertenso. Não foram encontradas diferenças estatísticas entre os grupos investigados para todas as características avaliadas.

Tabela 1 - Características antropométricas, cardiovasculares, medicamentosa e número de repetições de 1RM no grupo normotenso e hipertenso.

\begin{tabular}{lccc} 
& $\begin{array}{c}\text { GRUPO } \\
\text { NORMOTENSO }\end{array}$ & $\begin{array}{c}\text { GRUPO } \\
\text { HIPERTENSO }\end{array}$ & p \\
\hline FC basal $(\mathrm{bpm})$ & $(\mathrm{N}=08)$ & $(\mathrm{N}=12)$ & 0,693 \\
\hline PAS basal $(\mathrm{mmHg})$ & $76 \pm 2$ & $79 \pm 3$ & 0,051 \\
\hline PAD basal $(\mathrm{mmHg})$ & $128 \pm 2$ & $138 \pm 3$ & 0,760 \\
\hline PAM basal $(\mathrm{mmHg})$ & $80 \pm 2$ & $86 \pm 2$ & 0,304 \\
\hline LF basal un & $93 \pm 1$ & $95 \pm 3$ & 0,207 \\
\hline HF basal un & $47 \pm 3$ & $49 \pm 3$ & 0,137 \\
\hline BF/AF & $53 \pm 2$ & $51 \pm 3$ & 0,091 \\
\hline Medicamentos & $1,07 \pm 0,01$ & $1,14 \pm 0,11$ & \\
\hline 1 RM $($ Kg) & & Diuréticos $(n=4)$ IECA $(n=6)$ & 0,796 \\
\hline
\end{tabular}

Os dados estão apresentados com médias e erro padrão; F - feminino; $M$ - masculino; IMC - Índice de massa corporal; RM - repetição máxima; FC - Frequência Cardíaca; PAS - Pressão Arterial Sistólica; PAD - Pressão Arterial Diastólica; PAM - Pressão Arterial Média; IECA - inibidor da enzima conversora de angiotensina.

\section{Resposta da frequência cardíaca}

O protocolo EX40\% promoveu um aumento significativo da FC quando comparado com a condição de repouso, de $76 \pm 2 \mathrm{bpm}$ para $92 \pm 7 \mathrm{bpm}$ no grupo normotenso e de $79 \pm 3 \mathrm{bpm}$ para $93 \pm 5 \mathrm{bpm}$ no grupo hipertenso. Comportamento semelhante foi encontrado na intensidade EX60\%, tanto no grupo normotenso (de $76 \pm 2 \mathrm{bpm}$ para $93 \pm 8 \mathrm{bpm}$ ), quanto no grupo hipertenso (de $79 \pm 3 \mathrm{bpm}$ para $97 \pm 5 \mathrm{bpm}$ ). Os protocolos com intensidades de 40 e $60 \%$ de $1 \mathrm{RM}$ resultaram em aumentos similares da FC, no grupo de normotensos e hipertensos, e sem diferença estatística entre eles. O período de recuperação de 30 minutos após o exercício foi suficiente para restaurar a FC para valores próximos à condição de repouso nas duas intensidades do exercício. Estes dados podem ser observados na figura 1.

\section{Resposta da pressão arterial}

Os protocolos EX40\% e EX60\% promoveram aumento significativo apenas da Pressão Arterial Sistólica, em ambos os grupos, e sem diferença estatística entre os mesmos. Após os 30 minutos de recuperação, apenas PAS se apresentou reduzida 
em relação às condições basais, nos dois grupos e nos dois protocolos testados, sem diferenças estatísticas entre esses. Da condição basal para o final do período de recuperação, a redução da PAS do grupo de normotensos foi de $128 \pm 1$ para 108 $\pm 2 \mathrm{mmHg}$ no protocolo EX40\%, enquanto os hipertensos apresentaram redução de $138 \pm 3$ para $118 \pm 2 \mathrm{mmHg}$. $\mathrm{Na}$ intensidade EX60\%, o grupo normotenso apresentou redução de $128 \pm 1$ para $108 \pm 2 \mathrm{mmHg}$ e o grupo hipertenso de 138 \pm 3 para $117 \pm 3 \mathrm{mmHg}$. Com relação a Pressão Arterial Diastólica não foram encontradas modificações significativas entre as duas intensidades para os dois grupos, tanto durante, quanto após as sessões de exercício.

Figura 1 - Comportamento da frequência cardíaca nos momentos de repouso, durante e na recuperação do exercício, em hipertensas e normotensas, nas intensidades de 40 e $60 \%$ de 1 RM.

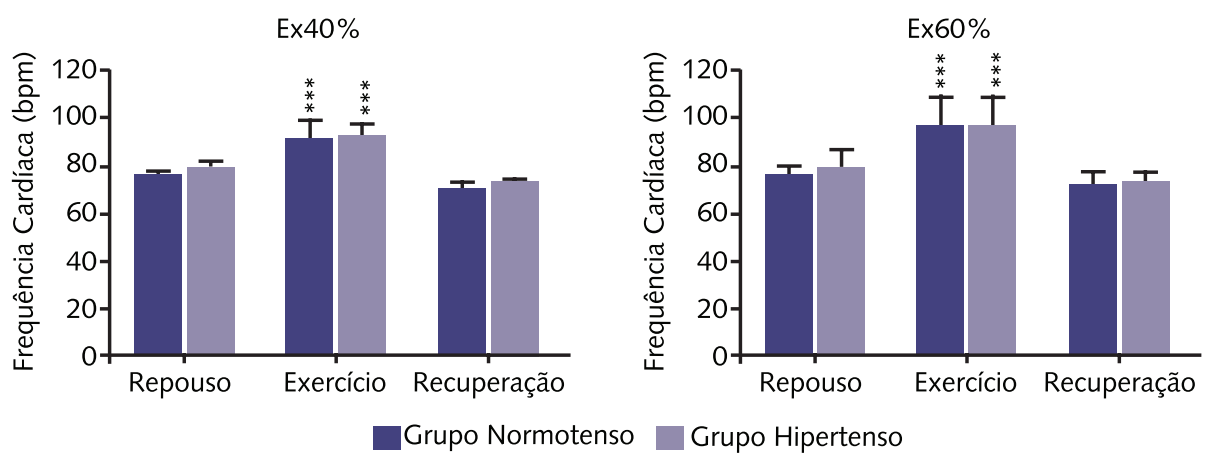

Os dados estão apresentados com média e desvio padrão da média; ${ }^{* *}$ significa $p<0,001$, comparado com repouso.

Figura 2 - Variação da pressão arterial sistólica e diastólica durante o exercício e no período de recuperação, nas intensidades de 40 e $60 \%$ de 1 RM, em normotensas e hipertensas.
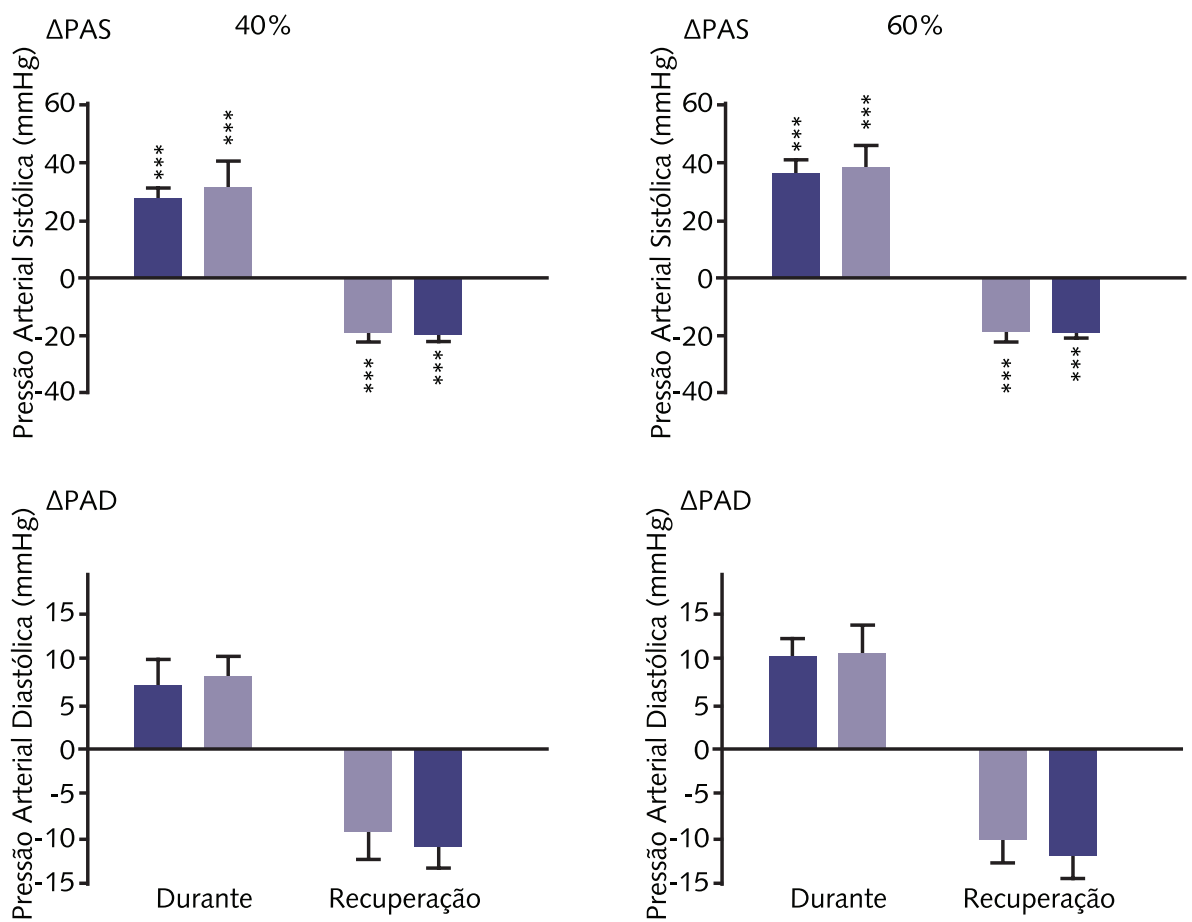

Grupo Normotenso

Grupo Hipertenso

Os dados estão apresentados com média e desvio padrão da média. ${ }^{* *}$ significa $p<0,01$ comparado com repouso. 


\section{Modulação autonômica cardíaca}

A modulação autonômica cardíaca no repouso, durante e após os protocolos de exercício estão apresentados na tabela 2. Os dois protocolos de exercício promoveram aumento significativo do componente $\mathrm{BF}$ e da razão $\mathrm{BF} / \mathrm{AF}$, e redução do componente AF tanto para as normotensas quanto para as hipertensas. Nenhuma diferença foi observada entre normotensas e hipertensas, seja para os componentes de $\mathrm{AF}$ e $\mathrm{BF}$ ou para a razão entre estes componentes em qualquer dos dois protocolos de exercício realizados.

No período de recuperação, os componentes BF, AF e BF/AF retornaram aos valores próximos aos basais em ambos os grupos no protocolo EX40\%. Em EX60\%, os valores de BF foram restabelecidos apenas para as hipertensas. Por outro lado, as normotensas apresentaram redução significativa em relação aos valores basais. O componente AF retornou a valores próximos aos basais em ambos os grupos. Como consequência as normotensas acabaram o protocolo EX60\% com $\mathrm{BF} / \mathrm{AF}$ maior que os hipertensos.

Tabela 2 - Modulação autonômica cardíaca no repouso, durante e após o exercício nas intensidades de $40 \%$ (EX40\%) e $60 \%$ (EX60\%) de 1RM nos grupos normotenso e hipertenso.

\begin{tabular}{lcc}
\hline & $\begin{array}{c}\text { Grupo } \\
\text { Normotenso }\end{array}$ & $\begin{array}{c}\text { Grupo } \\
\text { Hipertenso }\end{array}$ \\
\hline $\mathrm{BF}_{\mathrm{R}-\mathrm{R}}$ un & & $49 \pm 3$ \\
\hline Repouso & $47 \pm 3$ & $60 \pm 2^{*}$ \\
\hline EX40\% & $59 \pm 3^{*}$ & $64 \pm 3^{*}$ \\
\hline EX60\% & $60 \pm 3^{*}$ & $50 \pm 3$ \\
\hline PÓS 40\% & $51 \pm 3$ & $53 \pm 3$ \\
\hline PÓS 60\% & $41 \pm 3^{*} \# \S$ & $51 \pm 3$ \\
\hline AF ${ }_{R-R}$ un & & $39 \pm 2^{*}$ \\
\hline Repouso & $53 \pm 2$ & $36 \pm 2 *$ \\
\hline EX 40\% & $41 \pm 3^{*}$ & $49 \pm 3$ \\
\hline EX 60\% & $40 \pm 5^{*}$ & $49 \pm 4$ \\
\hline PÓS 40\% & $50 \pm 4$ & $1,14 \pm 0,11$ \\
\hline PÓS 60\% & $58 \pm 4 \# \S$ & $1,32 \pm 0,02^{*}$ \\
\hline BF/AF & & $1,33 \pm 0,03^{*}$ \\
\hline Repouso & $1,07 \pm 0,01$ & $1,10 \pm 0,16$ \\
\hline EX 40\% & $1,25 \pm 0,04^{*}$ & $1,05 \pm 0,21$ \\
\hline EX 60\% & $1,26 \pm 0,04^{*}$ & \\
\hline PÓS 40\% & $1,02 \pm 0,19$ & $0,91 \pm 0,11 \S$ \\
\hline PÓS 60\% & & \\
\hline
\end{tabular}

Os dados estão apresentados com média e desvio padrão da média; BF - Baixa Frequência; AF - Alta Frequência; BF/AF - Balanço Autonômico Cardíaco; R-R - Intervalo Cardíaco; un - unidade normalizada; PÓS $40 \%$ - após sessão a $40 \%$; PÓS $60 \%$ - após sessão a $60 \%$. * significa $p<0,01$, comparado com o repouso. \# significa $p<0,05$ comparado com $40 \%$ intragrupo. § significa $p<0,05$ comparado com grupo hipertenso.

\section{DISCUSSÃO}

\section{Respostas da frequência cardíaca e da atividade nervosa autonômica}

No nosso estudo, o aumento da FC durante o exercício foi similar entre as duas intensidades, ficando em torno de 16 e $18 \mathrm{bpm}$ para grupo de normotensos e hipertensos, respectivamente. Lamotte et al., ${ }^{14}$ encontraram aumentos de $20 \mathrm{bpm}$ 
durante uma sessão de exercício de intensidade moderada realizado na cadeira extensora com indivíduos cardiopatas. Esta elevação é considerada relativamente pequena, sugerindo que o ER de intensidade moderada não promove repercussões cronotrópicas importantes. Este comportamento da FC foi acompanhado pelo aumento do componente simpático, o que pode ser definido como um comportamento fisiologicamente esperado, uma vez que os sistemas cardiovascular, nervoso autonômico cardíaco e muscular interagem durante a realização do exercício ${ }^{18}$ fenômeno este que corrobora com dados prévios de Iellamo et al., ${ }^{19}$ para um exercício de extensão de joelho.

Aditivamente, a FC pós-exercício apresentou uma resposta satisfatória, uma vez que após os 30 minutos de recuperação ela se apresentou restaurada a valores próximos dos basais tanto nos hipertensos quanto nos normotensos. Este fenômeno foi acompanhado por restauração também do componente simpático. Em contraste com nossos dados, Rezk et al., ${ }^{10}$ e Queiroz et al., ${ }^{20}$, não encontraram redução da atividade nervosa autonômica simpática após 90 minutos de recuperação de protocolo de exercício com três series de quinze repetições e intensidade moderada realizado em jovens adultos normotensos. Uma justificativa para esse fenômeno pode ser o fato de que os protocolos de exercício destes autores contemplaram em torno de oito exercícios e para intensidade moderada em torno de 20 repetições, resultando em um maior volume de trabalho muscular. $\mathrm{O}$ fato de não ter sido encontrada diferença estatística entre os dois grupos estudados para a FC, bem como para a atividade autonômica cardíaca, indica que a resposta cronotrópica e nervosa autonômica não é influenciada pela presença de hipertensão em sujeitos idosos quando realizam um único ER com intensidade moderada.

Estudos prévios em que a variabilidade da FC foi investigada em sessões agudas de exercício resistido ${ }^{10,21}$ foram conduzidos com indivíduos jovens e saudáveis. Estes estudos apontam para ausência de aumento significativo na resposta autonômica cardíaca parassimpática tanto com sessão aguda de exercício, quanto com o treinamento. Assim, é notória a relevância de investigar o efeito do ER na modulação autonômica especificamente em grupos especiais como indivíduos com hipertensão. Com idosos, estudos ainda são escassos e conduzidos com protocolos de treinamento ${ }^{12,22}$, gerando consequentemente respostas crônicas diferentes, de modo que as respostas agudas ainda são desconhecidas. Até onde sabemos, o presente estudo foi o primeiro que avaliou a resposta autonômica cardíaca ao ER dinâmico em uma sessão aguda, realizado em idosas hipertensas.

Já se sabe que a hipertensão arterial, juntamente com o processo de envelhecimento estão associados à disfunção autonômica cardíaca, com aumento da atividade nervosa simpática e redução da resposta vagal, além de aumento na modulação simpática vasomotora ${ }^{23}$. Em nosso estudo foi observado que o componente de BF apresentou um aumento significativo nos hipertensos após EX60\%. Mesmo assim, esta resposta não representaria um risco cardiovascular para os indivíduos hipertensos, pois eles não apresentaram diferenças significativas em relação ao grupo normotenso. Além disso, um curto período de recuperação já se mostrou suficiente para restaurar sua atividade a valores próximos aos encontrados na condição basal.

\section{Resposta da pressão arterial}

Os indivíduos hipertensos e normotensos responderam às sessões de exercícios resistidos com comportamento fisiológico normal da PA, o que corrobora com vários estudos prévios ${ }^{22,24,25}$. Os aumentos da PAS na faixa de 30 a $40 \mathrm{mmHg}$ encontrado em ambos os grupos nos protocolos EX40\% e E60\%, são clinicamente 
seguros quando comparados com exercícios aeróbios. Além disso, não se relacionam com incidentes cardiovasculares durante o exercício ${ }^{26-27}$.

As idosas hipertensas e normotensas responderam aos dois protocolos de exercício resistido com significativa hipotensão sistólica pós-exercício. Embora a hipotensão diastólica não tenha sido significativa, a redução próxima de $10 \mathrm{mmHg}$ nos dois grupos e nos dois protocolos é clinicamente expressiva ${ }^{27}$. Mediano et al. ${ }^{28}$, demonstraram redução da PAS de aproximadamente $6 \mathrm{mmHg}$ e PAD de $2 \mathrm{mmHg}$, após uma sessão com cinco exercícios e três séries de dez repetições. Mota et al., ${ }^{25}$ encontraram HPE de aproximadamente 12 e $9 \mathrm{mmHg}$ para PAS e PAD respectivamente, após 45 minutos de recuperação de ER realizado com 13 exercícios, sendo uma série de 20 repetições à $40 \%$ de 1 RM. Apesar disso, o nosso traz a informação adicional de que com um volume tão pequeno, como três séries de um único exercício, já se pode obter redução pressórica importante em idosa normotensas e hipertensas.

Logo, a relevância deste estudo é que o ER de baixa e moderada intensidade pode ser considerado seguro para idosos hipertensos em comparação com congêneres normotensos. Uma vez que, nosso protocolo realizou apenas um exercício, esta recomendação deve ser avaliada, pois sessões de treinamento costumam ter maior volume de trabalho. Estudos posteriores devem levar em consideração o efeito somatório dos vários exercícios na sobrecarga hemodinâmica em uma mesma sessão de exercício.

\section{Limitações, contribuiç̧ões para a prática clinica e perspectivas futuras}

Diante do exposto, torna-se claro que é a escolha da intensidade de 40 ou $60 \%$ na elaboração de programas de exercício resistido, não parece fazer diferenças importantes do ponto de vista da solicitação cardíaca. Importante notar que esta premissa pode ser adotada mesmo tanto para sujeitos idosos e hipertensos. Logo, a relevância deste estudo é que o exercício resistido de baixa e moderada intensidade pode ser considerado seguro para idosos hipertensos em comparação com congêneres normotensos. Contudo, esta indicação deve ser avaliada pelo fato de que nosso protocolo realizou apenas um exercício, desde que as sessões de treinamento costumam ter maior volume de trabalho. Estudos posteriores devem levar em consideração o efeito somatório dos vários exercícios na sobrecarga hemodinâmica em uma mesma sessão de exercício. Nesse sentido, sugerimos como perspectivas futuras a realização de mais estudos com a avaliação da modulação autonômica cardíaca e outros mecanismos reguladores, após a realização de exercícios para membros inferiores e superiores, além de uma investigação aprofundada utilizando protocolos de exercícios com intensidades mais elevadas ou envolvendo números de repetições e intervalos diferenciados.

\section{CONCLUSÃO}

Diante dos resultados, podemos sugerir que o exercício resistido com intensidade moderada parece ser mais benéfico na prescrição de programas de exercício físico para idosas hipertensas, visto que sua resposta foi similar a aquela observada em idosas normotensas, além de não promover exacerbação da modulação autonômica cardíaca durante e após sua execução.

\section{REFERÊNCIAS}

1. Seals DR, Monahan KD, Bell C, Tanaka H, Jones PP. The aging cardiovascular system: changes in autonomic function at rest and in response to exercise. Int J Sport Nutr Exerc Metab. 2001; 11:189-95. 
2. Fallen EL. Vagal afferent stimulation as a cardioprotective strategy? Introducing the concept. Ann Noninvasive Electrocardiol. 2005; 10(4):441-6.

3. Lampert R. Anger and ventricular arrhythmias. Curr Opin Cardiol 2010; 25, (1):46-52,

4. De Meersman RE, Stein PK. Vagal modulation and aging. Biol Psychol. 2007; 74(2): 165-73.

5. Borst SE. Interventions for sarcopenia and muscle weakness in older people. Age Ageing, 2004; 33(6);548-555.

6. Dipietro L, Yeckel CW, Dziura J. Progressive improvement in glucose tolerance following lower-intensity resistance versus moderate-intensity aerobic training in older women. J Phys Act Health. 2008; 5(6):854-869.

7. Melo CM, Alencar Filho AC, Tinucci T, Mion D.Jr, Forjaz C. Postexercise hypotension induced by low-intensity resistance exercise in hypertensive women receiving captopril. Blood Press Monit. 2006; 11(4):183-9.

8. Nelson ME, Rejeski WJ, Blair SN, Duncan PW, Judge JO, King AC, Macera CA, CastanedaSceppa C. Physical activity and public health in older adults: recommendation from the American College of Sports Medicine and the American Heart Association. Circulation. 2007; 116(9): p.1094-1105.

9. Paterson DH, Jones GR, Rice CL. Aging and physical activity data on which to base recommendations for exercise in older adults. Appl Physiol Nutr Metab. 2007; 32(2):75-171.

10. Rezk CC,Marrache RC,Tinucci T, Mion DJr, Forjaz CL. Post-resistance exercise hypotension, hemodynamics, and heart rate variability: influence of exercise intensity. Eur J Appl Physiol. 2006; 98(1):105-112.

11. Collier SR, Kanaley JA, Carhart RJr, Frechette V, Tobin MM, Bennett N, Luckenbaugh AN, Fernhall B. Cardiac autonomic function and baroreflex changes following 4 weeks of resistance versus aerobic training in individuals with pre-hypertension. Acta Physiolog (Oxf), 2009; 195(3):339-348.

12. Takahashi AC, Melo RC, Quiterio RJ, Silva E, Catai AM. The effect of eccentric strength training on heart rate and on its variability during isometric exercise in healthy older men. Eur J Appl Physiol. 2009; 105(2):315-323.

13. VI Diretrizes Brasileiras de Hipertensão Arterial. Sociedade de Cardiologia do Estado de São Paulo, Campos do Jordão, 2010.

14. Lamotte M, Niset G, Van De Borne P. The effect of different intensity modalities of resistance training on beat-to-beat blood pressure in cardiac patients. Eur J Cardiovasc Prev Rehabil. 2005; 12(1):12-7.

15. Kraemer WJ, Fry AC. Strength testing: development and evaluation of methodology. In: Maud PJ, Foster C, eds. Physiological assessment of human Wtness. Champaign: Human Kinetics. 1995:115-138.

16. Malliani A, Pagani M, Lombardi F, Cerutt S. Cardiovascular neural regulation explored in the frequency domain. Circulation. 1991; 84:482-492.

17. Heart rate variability. Standards of measurement, physiological interpretation, and clinical use. Task Force of the European Society of Cardiology and the North American Society of Pacing and Electrophysiology. Eur Heart J. 1996; 17(3):354-381.

18. Ray CA, Wilson TE. Comparison of skin sympathetic nerve responses to isometric arm and leg exercise. J Appl Physiol. 2004; 97(1):160-164.

19. Iellamo F, Pizzinelli P, Massaro M, Raimondi G, Peruzzi G, Legramante JM. Muscle metaboreflex contribution to sinus node regulation during static exercise: insights from spectral analysis of heart rate variability.Circulation. , 1999; 100(1):27-32.

20. Queiroz AC, Gagliardi JF, Forjaz CLM, Rezk CC. Clinic and ambulatory blood pressure responses after resistance exercise. J Strength Cond Res. 2009; 23(2): 571-578.

21. Heffernan KS, Kelly EE, Collier SR, Fernhalla B. Cardiac autonomic modulation during recovery from acute endurance versus resistance exercise. Eur J Prev Cardiol. 2006;13:80-86.

22. Melo RC, Quiterio RJ, Takahashi AC, Silva E, Martins LE, Catai AM. High eccentric strength training reduces heart rate variability in healthy older men. Br J Sports Med. 2008; 42(1):59-63.

23. Pagani M, Lucini D. Autonomic dysregulation in essential hypertension: insight from heart rate and arterial pressure variability. Auton Neurosci. 2001; 90: 76-82.

24. Fisher MM. The effect of resistance exercise on recovery blood pressure invnormotensive and borderline hypertensive women. J Strength Cond Res. 2001; 15(2):210-216. 
25. Mota MR, Pardono E, Lima LC, Arsa G, Bottaro M, Campbell CS, Simões HG. Effects of treadmill running and resistance exercises on lowering blood pressure during the daily work of hypertensive subjects. J Strength Cond Res. 2005, 23(8):2331-2338.

26. Pollock ML, Franklin BA, Balady GJ, Chaitman BL, Fleg JL, Fletcher B, Limacher M, Piña IL, Stein RA, Williams M, Bazzarre T. AHA Science Advisory. Resistance exercise in individuals with and without cardiovascular disease: benefits, rationale, safety, and prescription: An advisory from the Committee on Exercise, Rehabilitation, and Prevention, Council on Clinical Cardiology, American Heart Association; Position paper endorsed by the American College of Sports Medicine. Circulation. 2000; 101(7): 828-833.

27. Williams MA, Haskell WL, Ades PA, Amsterdam EA, Bittner V, Franklin BA, Gulanick M, Laing ST, Stewart KJ. American Heart Association Council on Clinical Cardiology; American Heart Association Council on Nutrition, Physical Activity, and Metabolism. Resistance exercise in individuals with and without cardiovascular disease: 2007 update: a scientific statement from the American Heart Association Council on Clinical Cardiology and Council on Nutrition, Physical Activity, and Metabolism. Circulation, 2007; 116 (5):572-584.

28. Mediano MFF, Paravidino V, Simão R, Pontes FL, Polito MD. Comportamento subagudo da pressão arterial após o treinamento de força em hipertensos controlados. Rev Brasil de Med do Esport. 2005; 11(6):337-340.

$$
\begin{array}{r}
\text { Endereço para Correspondência } \\
\text { Aline de Freitas Brito } \\
\text { Universidade Federal da Paraíba - Cen- } \\
\text { tro de Ciências da Saúde/ Departamen- } \\
\text { to de Educação Física; Bairro: Cidade } \\
\text { Universitária; Cidade: João Pessoa/PB. } \\
\text { CEP: 58051900 } \\
\text { Telefone: (83) } 3216-7200 \\
\text { e-mail: enylla_sophia@hotmail.com }
\end{array}
$$

Recebido 20/09/2013

Revisado 09/10/2013

Aprovado 09/10/2013 